\title{
Isofemale lines in Drosophila: an empirical approach to quantitative trait analysis in natural populations
}

\author{
JR David, P Gibert, H Legout, G Pétavy, P Capy and B Moreteau \\ CNRS, UPR 9034, Laboratoire Populations, Génétique et Evolution, Bât. 13, 91198 Gif sur Yvette Cedex, France
}

\begin{abstract}
Founding isofemale lines from wild collected females is a basic tool for investigating the genetic architecture of Drosophila natural populations. The method permits the analysis of quantitative traits under laboratory conditions, with a much broader scope than the mere evidence of a significant genetic heterogeneity among lines. Genetic variability is generally demonstrated by a significant coefficient of intraclass correlation, but several experimental precautions are needed and explained here. The relationship between classical (additive) heritability and intraclass correlation is not straightforward, presumably because the genetic bottlenecks due to the initiation of the lines unravel a significant, nonadditive genetic variance due to dominance and epistatic effects. It is thus suggested to consider intraclass correlation as a specific genetic parameter that enables comparisons between different traits, different populations or different environments. The use of isofemale lines is, however, not restricted to the calculation of an
\end{abstract}

intraclass correlation. It can be used to estimate genetic correlations among traits or environments. The method is also convenient for the analysis of phenotypic plasticity in relation to an environmental gradient. A precise description of the response curves (the reaction norms) is possible, distinguishing trait parameters and plasticity parameters. A fairly general conclusion is that, for a given trait, mean value and plasticity are genetically independent. It is also possible to analyze traits, which, like sexual dimorphism, must be measured on different individuals, and even to demonstrate their genetic variability. In many cases, further empirical and theoretical analyses are possible and needed. It is argued that, in the future, isofemale lines will have an increasing significance among the various techniques appropriate to the analysis of quantitative evolutionary genetics in a diversity of species.

Heredity (2005) 94, 3-12. doi:10.1038/sj.hdy.6800562

Published online 25 August 2004

Keywords: isofemale lines; Drosophila melanogaster, natural populations; intraclass correlation; genetic correlation; phenotypic plasticity; ecological studies

\section{Introduction; sampling natural populations}

Drosophila melanogaster has proved a remarkable laboratory model for molecular and developmental genetic investigations. It is also an excellent material for ecological and evolutionary genetic studies. Indeed, its natural populations are highly differentiated spatially (David and Capy, 1988) and a diversity of geographic patterns have been observed. Short distance variations have been observed in various parts of the world, generally in relation with alcoholic resources and adult behavioral preferences (Karan et al, 1999b; Haerty et al, 2003). Long distance variations may be related to diffusion processes (Gockel et al, 2001), such as a longitudinal trend (Capy et al, 1994; Aulard et al, 2002). However, the most frequent variations are related to latitude, they concern a diversity of traits including morphology, physiology and behaviour, and the strong correlation with temperature strongly suggests that local

Correspondence: JR David, CNRS, UPR 9034, Laboratoire Populations, Génétique et Evolution, Bât. 13, 91198 Gif sur Yvette Cedex, France.

E-mail:david@pge.cnrs-gif.fr

Accepted 28 June 2004; published online 25 August 2004 climatic variations are the main selective factors (David et al, 1983; 2004a; David and Capy, 1988; Gibert et al, 2004).

Analyzing the genetic architecture of a natural population raises a major problem: how can one get an unbiased sample of wild living flies? A possible strategy is to collect several (many) inseminated females and use them to establish a laboratory mass culture. Most traditional laboratory strains, such as Oregon or Canton, were established in that way. A better methodology is to isolate the wild collected females in separate culture vials, to initiate what might be a full sib family, and what, after Parsons and Hosgood (1967) is traditionally called an isofemale line. There are at least two difficulties raised by such a procedure.

Firstly, it is now known that most wild caught females have been inseminated by several males (Milkman and Zeitler, 1974; Imhof et al, 1998) and there are two ways to overcome this problem. One is to cross one female and one male from two independent lines and, in that way, generate a 'new line' which harbors only four independent haploid genomes (see Capy et al, 1993). A second method is to isolate in each culture vial one wild caught female and one wild caught male, and to collect the 
progeny in a fresh vial after a few days. Owing to the precedence of the last male sperm (Simmons, 2001), almost all progeny will be sired by this last male.

The second difficulty is the spatial structure of the sampled natural population. If the wild living flies are collected in a single trap, they are likely to be genetically related (eg progeny from the same mother), so that a given chromosome will be sampled more than once. Ideally, a 'local' natural population should be sampled by using a fairly large number of distant traps. Surprisingly, this problem is rarely addressed in publications. A similar but even stronger bias is expected when isofemale lines are founded from the adults emerging from a trap deposited in nature.

Isofemale lines can be used for providing two kinds of genetic information. If genetic markers are analyzed, lines can be frozen and kept for eventual investigations. If live material is needed, for example for cytogenetic studies, the lines can be kept alive for many generations. As a general principle, a single fly from each line should be used in any genetic characterization. In this respect, a question which frequently arises is: how many lines and how many individuals per line have to be analyzed to get a representative sample of a population? A solution was proposed by Capy and Rouault (1987), based on a sampling/resampling method, and using a multinomial approach. On the one hand, it was shown that for estimating the allelic frequency at a given locus only two individuals per line were sufficient. On the other hand, the number of lines needed depended on the number of alleles and on their respective frequencies.

Things become more difficult when quantitative traits, be they morphometry, physiology or behavior, are investigated. Such traits are generally more directly related to adaptation and, as pointed out by numerous investigators (eg Lewontin, 1974) are more interesting to evolutionary geneticists than are molecular markers. They are, however, more difficult to quantify, since experimental conditions, such as temperature and food quality, must be precisely defined and may change the mean values of quantitative characters. Also, such traits, when measured on laboratory isofemale lines, are likely to change over successive generations, but in two opposite ways. On the one hand, adaptation to laboratory conditions should reduce the variance between lines, but also may progressively modify the overall mean. On the other hand, genetic drift among lines is expected to produce more and more divergent lines in the course of generations.

The aim of this paper is to show that, in spite of many problems and possible pitfalls, the isofemale line technique (ILT) remains a central method for characterizing quantitative traits in a natural population. Various possibilities will be illustrated here, using $D$. melanogaster data. It is not only a means to investigate the genetic variability harbored by a natural population, as shown by Hoffmann and Parsons (1988), but a general technique which permits the analysis of a diversity of biological problems related to ecological genetics and evolutionary genetics.

\section{Data structure for quantitative traits}

A set of lines is isolated from a natural population, the progeny are grown under controlled, although arbitrarily chosen, conditions (food and temperature), and a given number $(n)$ of flies from each line is measured for quantitative traits. Each line will be characterized by the mean value and the variance of the trait.

As an illustration, we give (Figure 1) the distributions of wing and thorax length in two samples of 10 lines from a European temperate population and an Afrotropical population. Such populations are known to correspond to the ends of a well-known latitudinal cline (Capy et al, 1993), and we see that there is no overlap between the distributions of the line means. There is of course a broader variability, and a slight overlap, when the individual flies are considered (Figure 1). A one-way ANOVA (Table 1) shows that there are two highly significant effects, one due to populations, the other due to lines (nested in populations). The interpretation of the population effect is straightforward: tropical flies are smaller than temperate ones. It is to be noted that, in this case, a significant difference would also be observed if only two sets of values, from two mass cultures, were
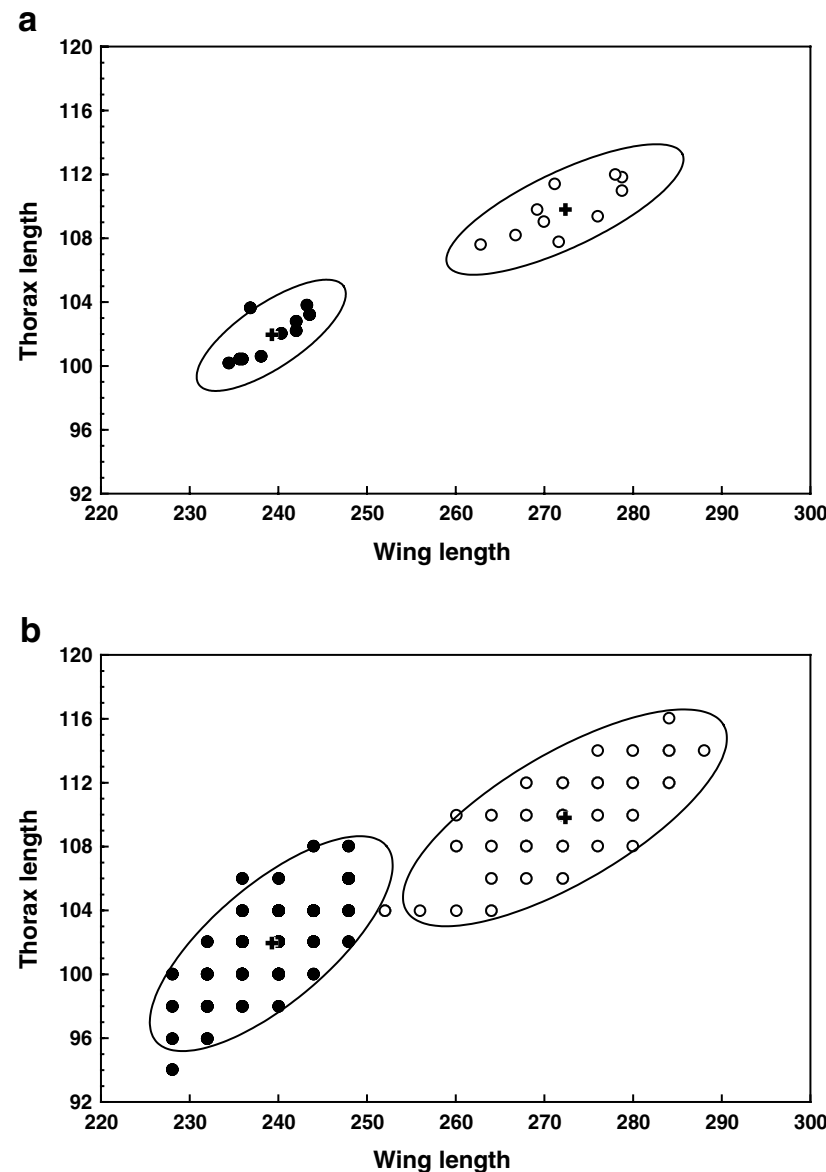

Figure 1 Scatterplot of female wing and thorax length values in two natural populations of D. melanogaster from tropical Africa (Sao Tomé, filled circles) and France (Bordeaux, open circles). Ellipses of $90 \%$ probability are shown to help visualize the distributions. (a) Distribution of mean values of isofemale lines $(n=10$ flies in each line) in the two geographic populations. Notice that, for wing length, the difference between the most extreme lines from France and Africa exceeds 8 standard deviations. (b) Distribution of individual values ( $n=100$ in each case). Notice that the number of dots is less, due to many identical values. Lengths are expressed in $\mathrm{mm} * 100$. 
Table 1 Results of ANOVA on the data shown in Figure 1

\begin{tabular}{|c|c|c|c|c|c|c|c|c|c|}
\hline \multirow[t]{2}{*}{ Source } & \multirow[t]{2}{*}{$d f$} & \multicolumn{4}{|c|}{ Wing length } & \multicolumn{4}{|c|}{ Thorax length } \\
\hline & & MS & $\mathrm{F}$ & $\mathrm{P}$ & Variation explained (\%) & MS & $\mathrm{F}$ & $\mathrm{P}$ & Variation explained (\%) \\
\hline Population & 1 & 54846.7 & 264.2 & $* * *$ & 87.0 & 3104.72 & 129.3 & $* * *$ & 63.4 \\
\hline Line (pop) & 18 & 207.61 & 8.407 & $* * *$ & 5.93 & 24.009 & 4.308 & $* * *$ & 9.52 \\
\hline Individuals & 180 & 24.693 & & & 7.05 & 5.573 & & & 22.1 \\
\hline
\end{tabular}

df: degree of freedom; MS: mean square; $F$ : variance ratio; $P$ : probability of significance: ${ }^{* * *} P<0.001$.

available. The advantage of the ILT is to produce more convincing results showing that any wild caught female would be correctly assigned to its origin population by looking at its laboratory grown progeny.

The significant heterogeneity among lines is more difficult to interpret. It is traditionally assumed that it has a genetic basis (Hoffmann and Parsons, 1988; Capy et al, 1994), but this is just an hypothesis. In the following section, we discuss this point in more detail.

\section{Variability among lines: arguments and proofs for a genetic basis}

Although grown at the same time at a controlled temperature and on the same batch of Drosophila medium, line progeny are grown in different vials, each of which is a special environment. The number of larvae may be variable and, more importantly, different microorganisms (molds, bacteria) may develop in different vials. In other words, differences among lines can be due to heterogeneity among vials, that is, a common environment effect (CEE). Several approaches may be used to analyze this problem and minimize its significance.

Ideally, only a single fly should be taken from a given vial, so that CEEs (if any) would be included in the individual variability. This technique is, however, very time (and vial) consuming and is rarely implemented (eg Robinson and Partridge, 2001). An intermediate solution is to grow each line in two or more vials and measure flies from several vials. An ANOVA is then undertaken to look for a significant vial effect (nested within lines). Such significant effects may be observed (Hoffmann and Parsons, 1988; Imasheva et al, 1998, 1999) and complicate, or even prevent, further analyses.

A significant vial effect is an argument against a genetic basis for among-line variation. It is, however, possible to design positive methods to demonstrate a genetic component. At least two such techniques have been implemented.

Moreteau et al (1995) compared isofemale lines with what were called isogroups. From a natural population, single pairs were isolated to produce classical isofemale lines; simultaneously, groups of 10 pairs were created and allowed to oviposit in a vial. A total of 10 progeny from each line or group were measured. The assumption was that, if CEEs were preponderant, the variances among lines and among groups would be similar. If, on the contrary, variation among lines had a genetic basis, each isogroup would be akin to a mass population and variation among them should not be significant. The results, illustrated in Figure 2, confirmed the second
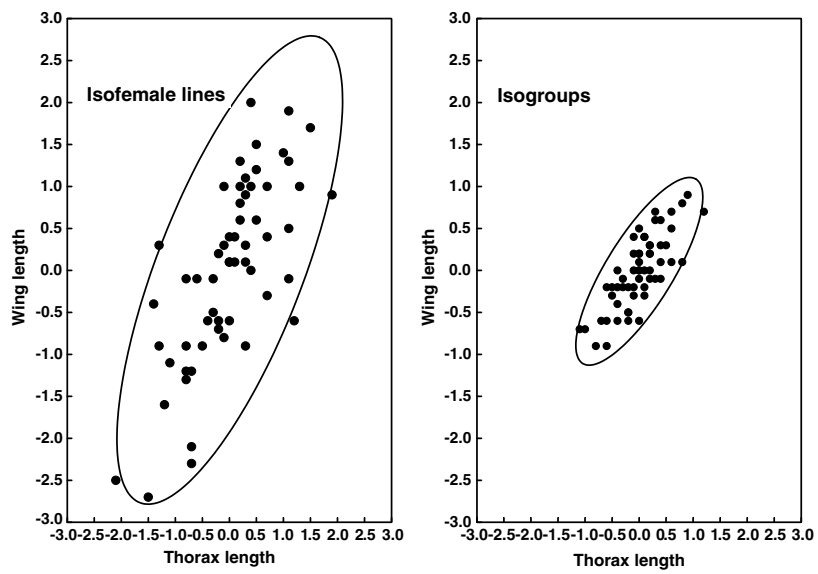

Figure 2 Comparison of the variability observed between isofemale lines and similar isogroups (from Moreteau et al, 1995). Isofemale lines are the progeny of single wild collected females, while isogroups are the progeny of 10 wild collected pairs in the first laboratory generation. Since different samples from different populations with different mean values were available, we show here the deviations from the overall mean of each population. Each point corresponds to the mean thorax or wing length of 10 females.

expectation: the variability among isogroups was always much less than that among lines. ANOVA, however, revealed a general trend for a slight but significant heterogeneity among groups. This might be the consequence of some CEE. However, it could also reflect the fact that the effective size of the parental groups was certainly much less than 10 pairs. We know that natural populations exhibit a huge phenotypic variability (Coyne and Beecham, 1987; Imasheva et al, 1994; Moreteau et al, 1995; Gibert et al, 1998a) and that smaller females will oviposit very few eggs (Boulétreau, 1978), while smaller males will leave less progeny (Partridge et al, 1987). If, instead of 20, the effective average size of each group was 3 or 4 times less, significant genetic differences would still appear among their progeny.

Another convincing method to demonstrate a genetic basis to isofemale line variations is to investigate their mean values in different generations (Krebs and Loeschcke, 1997; Gibert et al, 1998a; Karan et al, 1998). Such experiments have shown a positive correlation between mean values in different generations. For example, the average between-generation correlation was $r=0.55 \pm 0.04$ for wing length and $r=0.84 \pm 0.01$ for abdomen pigmentation (Gibert et al, 1998a). The phenotype of a line is repeatable in different generations, and this phenomenon was called genetic repeatability (Gibert et al, 1998a). The value of the correlation is, 
however, influenced by the number of flies measured in each line, and presumably also by the sampling error in the choice of the parents at each generation. Genetic repeatability is not an estimate of heritability, although we expect that highly heritable traits will be more repeatable. This problem deserves more extensive empirical and theoretical investigations.

\section{Variability among lines: practical conclusions and advices}

The ILT has been implemented for the analysis of many traits, and from the results it has been generally concluded that the original population harbored significant genetic heterogeneity, which is revealed by this technique. While such a conclusion is generally correct, there are some caveats, and here we give practical advice for dealing with these.

The phenotypic variability is highly variable among different traits. As a rule, morphometrical traits such as body size, pigmentation, bristle number or ovariole number are only weakly sensitive to uncontrolled accidental variation in the experimental conditions. Consequently, CEEs for these traits are small and often can be neglected. Physiological or behavioral traits are, however, far more difficult to standardize, and more sensitive to accidental variation. This does not mean that such traits are not genetically repeatable, and indeed repeatability has been demonstrated for heat tolerance (Krebs and Loeschcke, 1997) or sexual behavior (Ritchie and Kyriacou, 1994). Other traits may be extremely variable under the same laboratory conditions. For example, measuring the recovery time from chill coma after a treatment at $0^{\circ} \mathrm{C}$ is an excellent means for distinguishing temperate (cold-tolerant) and tropical (cold-sensitive) species (Gibert et al, 2001). However, when measured on the same mass population under the same conditions, the recovery time proved to be highly variable, although the genetic make-up remained the same (David et al, 1997). The ILT applied to this trait would certainly reveal highly significant differences between lines, although the true genetic component could be very low.

Optimal rearing conditions favor and may be a prerequisite for a high genetic repeatability. For our morphometrical analyses, we use a high nutrient medium containing about $8 \%$ of dried yeast, in which microorganism development is prevented by a large amount of nipagine (David and Clavel, 1965). A single vial may produce several hundred normal looking flies, the size of which is practically independent of larval density (Karan et al, 1999a). By contrast, an ordinary sugar cornmeal medium, seeded with live yeast, is far more sensitive to crowding effects, and smaller flies are observed if the density reaches 100 per vial. Using the same food but not seeded with live yeast provides very poor results, specifically slow growth and small flies (unpublished results). The fact that different laboratories, investigating the same trait, do not use the same food, complicates all comparisons. As a rule, a nonoptimum medium always results in an increase of the variability at the individual level (see Gibert et al, 1998a). What occurs at the between-line level remains to be investigated.

\section{Variance estimates and intraclass correlation}

Under the above-mentioned conditions (a trait weakly sensitive to CEE, good feeding conditions, precise incubator), significant variations among lines do reveal genetic variability. ANOVA will produce a within line $(\mathrm{Vw})$ and a between line $(\mathrm{Vb})$ component. We consider here a sample of 30 lines from the same population and illustrate the results for wing and thorax length of both sexes (Table 2). $\mathrm{Vb}$ is a convenient estimate of the genetic variance $(\mathrm{Vg})$ when $n$ is sufficient $(n>20)$. If not, the following correction is suggested: $\mathrm{Vg}=\mathrm{Vb}-\mathrm{Vw} / n$.

With an isofemale line design, the genetic variability can be estimated by calculating the coefficient of intraclass correlation, $t$ (Hoffmann and Parsons 1988; Falconer 1989) according to the formula:

$$
t=\frac{\mathrm{Vb}-\mathrm{Vw} / n}{\mathrm{Vb}+(n-1) \mathrm{Vw} / n}=\frac{n \mathrm{Vb}-\mathrm{Vw}}{n \mathrm{Vb}+(n-1) \mathrm{Vw}}
$$

Values of $t$ are given in Table 2 .

An interesting, although not resolved, problem, is whether the ILT permits an estimate of the environmental, nongenetic variance, Ve. In this respect, several points of view may be considered. Ecologists often consider that the within-line variance harbors mainly a nongenetic component (Bell, 1997). In other words, $\mathrm{Vw} \approx \mathrm{Ve}$. However, quantitative genetic models (Falconer, 1989) show that Vw still harbors a significant genetic component: $\mathrm{Vw}>\mathrm{Ve}$. Finally, we could try to estimate Ve using the total phenotypic variance $\mathrm{Ve}=\mathrm{Vp}-\mathrm{Vg}$. The fact that $\mathrm{Vb}$ and $\mathrm{Vw}$ may vary in opposite ways was recently demonstrated (Pétavy et al, 2004) in lines submitted to a daily thermal stress. Both a cold and heat stress increased the within (environmental) variance, but opposite results were obtained for the between-line (genetic) variance: an increase following the cold stress but a decrease following the heat stress. More extensive investigations are needed concerning the significance of the two levels of variability.

\section{Intraclass correlation as an empirical, special estimate of genetic variability}

In quantitative genetics, heritability $\left(h^{2}\right)$ estimates the amount of additive genetic variability which is trans-

Table 2 Statistical parameters of wing and thorax length estimated on a sample of 30 isofemale lines from a French (Bordeaux) population

\begin{tabular}{lcclcc}
\hline & \multicolumn{2}{c}{ Wing length } & & \multicolumn{2}{c}{ Thorax length } \\
\cline { 2 - 3 } \cline { 5 - 6 } & Female & Male & & Female & Male \\
\hline Mean & $270.89 \pm 1.25$ & $233.37 \pm 1.40$ & & $109.03 \pm 0.52$ & $94.89 \pm 0.54$ \\
Vp & 53.19 & 42.30 & & 6.89 & 5.59 \\
$\mathrm{Vw}$ & 25.14 & 23.03 & & 4.21 & 3.80 \\
$\mathrm{Vb}$ & 30.57 & 21.60 & & 3.10 & 2.56 \\
$\mathrm{Vg}$ & 28.05 & 19.27 & & 2.68 & 2.18 \\
$\mathrm{CVg}$ & 1.96 & 1.88 & & 1.50 & 1.56 \\
$t$ & 0.53 & 0.46 & & 0.39 & 0.36 \\
\hline
\end{tabular}

Mean: expressed in $m m * 100$. Standard error $( \pm)$ calculated from the means of 30 lines; Vp: total phenotypic variance; Vw: within line variance; $\mathrm{Vb}$ : between line variance; $\mathrm{Vg}$ : estimate of genetic variance; CVg: genetic coefficient of variation (evolvability); $t$ : intraclass correlation. 
mitted from parents to offspring. Classical estimates can be carried out by several methods, including half-sib design, parent-offspring regression or directional selection. It is also generally assumed (Falconer 1989) that $t=0.5 h^{2}$. Indeed, Hoffmann and Parsons (1988) developed a more complex equation:

$$
h^{2}=\frac{2}{\left(\frac{1}{t}-0.5\right)}
$$

which gives a still higher value for $h^{2}$. Such calculations, applied to the data in Table 2, would provide some values of $h^{2}$ approaching or passing 1, which is meaningless. Trying to estimate $h^{2}$ from $t$ raises at least two problems. One is that, ideally, $t$ should be measured on the first laboratory generation among individuals which are full-sibs, but noninbred. Inbreeding occurs over successive laboratory generations and should increase the between line variance, due to genetic drift (see Robertson, 1952). An investigation over nine generations (Gibert et al, 1998a), however, revealed only a very small increase in $\mathrm{Vg}$.

The second and major problem is that $\mathrm{Vg}$, as measured by the ILT, is not the additive genetic variance, Vga, but is likely to include dominance and epistatic effects. Isolating isofemale lines from a natural population is an experimental approach for investigating the consequence of the strongest possible demographic bottleneck, followed by a population expansion. Theoretical models have shown that in such a case the genetic variability could be increased significantly, due to dominance and epistatic components being incorporated into the additive variance (Meffert, 2000). Although generally neglected in quantitative genetics studies, the role of epistatic interactions in evolutionary genetics is appearing more and more important (Wolf et al, 2000; Rice, 2002). Our hypothesis is that natural populations of $D$. melanogaster harbor a large amount of hidden epistatic interactions, which are revealed by a founder effect in isofemale lines. In other words, $t$ is generally closer to $h^{2}$ than $0.5 h^{2}$.

Empirical data favor this hypothesis. Numerous independent $h^{2}$ estimates have been made on morphometrical traits in D. melanogaster and its sibling $D$. simulans, and data compiled by Roff and Mousseau (1987). Similarly, intraclass correlations have been measured in many natural populations of the two species (Capy et al, 1994) and the results are compared in Table 3. If we calculate, for each trait, the $t / h^{2}$ ratio, the average value is $0.95 \pm 0.15(n=5)$. Clearly $t$ seems closer to $h^{2}$ than to $0.5 h^{2}$. Further investigations (eg a diallele design) remain to be carried out on natural populations of $D$. melanogaster for estimating dominance and epistatic components in the total genetic variance. For the moment, we suggest that investigations using isofemale lines should provide $t$ values as a demonstration of a genetic variability in the population of origin, which should not be transformed into $h^{2}$.

\section{Genetic correlations between different traits}

Variability between family means has mainly a genetic basis and ILT offers the advantage that different traits can be measured on the same line, whether or not measurements are carried out on the same flies. The correlation between family means is an estimate of a genetic correlation (Via, 1984; Gibert et al, 1998b). When $n$ (the family size) is small, a correction for estimating variances and covariances might be done as follows:

$$
r=\frac{\mathrm{CoVb} x y-1 / n \mathrm{CoVw} x y}{\sqrt{(\mathrm{Vb} x-l / n \mathrm{Vw} x(\mathrm{Vb} y-l / n \mathrm{Vw} y)}}
$$

where $\mathrm{CoVb}$ and $\mathrm{CoVw}$ are the between- and within-line covariances of $x$ and $y$, while $\mathrm{Vb}$ and $\mathrm{Vw}$ are the betweenand within-line variance of each variable, respectively.

The practical consequence of such corrections was analyzed on a large set of data on abdomen pigmentation, with $n=10$ (Gibert et al, 1998b). The general conclusion was straightforward. Corrected values were more variable, with the disadvantage of producing a significant proportion of values outside the range of a correlation coefficient, that is greater than 1 . So the advice from empirical studies is that genetic correlations should be estimated directly from family means, avoiding any correction for $n$.

Some quantitative traits provide more or less the same information and are positively correlated. For example, wing and thorax length are both size-related traits and are always positively correlated $(r \approx 0.70)$ (David et al, 1994) (see also Figures 1 and 2). Other quantitative traits are independent because their genetic bases are different. For example, there is no correlation between ovariole number and sternopleural bristle number (Moreteau et al, 2003). A positive correlation would, however, appear if different geographic populations are compared. This is due to the fact that both ovariole and sternopleural bristle numbers exhibit parallel latitudinal clines (Capy

\begin{tabular}{|c|c|c|c|c|c|}
\hline \multirow[t]{2}{*}{ Species } & \multirow[t]{2}{*}{ Trait } & \multicolumn{2}{|c|}{ Heritability ${ }^{a}$} & \multicolumn{2}{|c|}{ Intraclass correlation ${ }^{\mathrm{b}}$} \\
\hline & & $\mathrm{m} \pm s e$ & $\mathrm{n}$ & $\mathrm{m} \pm s e$ & $\mathrm{~N}$ \\
\hline \multicolumn{6}{|c|}{ D. melanogaster } \\
\hline & Wing length & $0.325 \pm 0.014$ & 66 & $0.403 \pm 0.021$ & 55 \\
\hline & Thorax length & $0.319 \pm 0.021$ & 30 & $0.232 \pm 0.019$ & 55 \\
\hline & Sternopleural bristles & $0.436 \pm 0.014$ & 87 & $0.295 \pm 0.017$ & 55 \\
\hline & Abdominal bristles & $0.415 \pm 0.026$ & 43 & $0.292 \pm 0.018$ & 55 \\
\hline D. simulans & Wing length & $0.297 \pm 0.012$ & 24 & $0.416 \pm 0.034$ & 25 \\
\hline
\end{tabular}

Table 3 Comparison of heritability and intraclass correlation for various quantitative traits in D. melanogaster and D. simulans

$m$ : mean \pm standard error; $n$ : number of independent estimates.

${ }^{a}$ Data from Roff and Mousseau (1987).

bData from Capy et al (1994). 


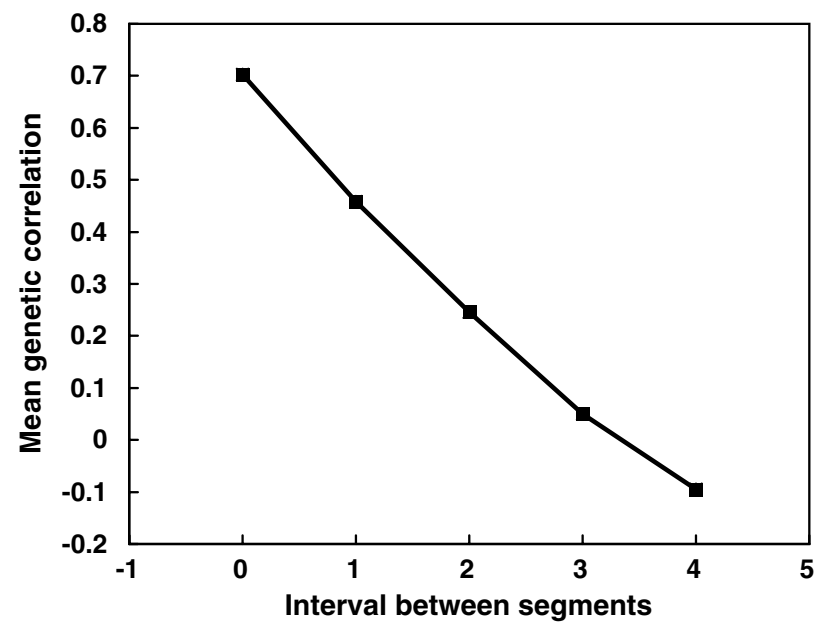

Figure 3 Analysis of genetic correlations of female abdomen pigmentation among successive segments (from Gibert et al, 2000). Genetic correlation is high when adjacent segments (eg 2-3 or 6-7) are compared. The correlation decreases almost linearly when more distant segments are compared, and becomes null when the interval reaches 3 (eg correlation between 2 and 6 or 3 and 7 ).

et al, 1993). Owing to the lack of intrapopulation correlation, we must conclude that both clines are due to natural selection, which however acts independently on the two traits.

Genetic correlations are especially interesting when considering a trait that is expressed in homologous, modular parts of the body. The best example is provided by pigmentation of abdominal segments $2-7$ in females (Gibert et al, 2000). Each segment has the same architecture: a sclerified, dorsal tergite and a membranous sternite. Each tergite harbors a dark strip at its posterior margin, the extension of which is genetically variable. From this observation, we expect that the same pigmentation genes are expressed in the different segments of the same fly, resulting in a high positive correlation among segments. Experimental results, illustrated in Figure 3, were more complex: a clear correlation (around 0.70) was found when any two successive segments were compared, but the correlation decreased regularly when more distant segments were considered, falling to zero when comparing segments 2 and 7. In other words, in spite of analogous phenotypes, different pigmentation genes are acting in the anterior and posterior abdomen segments.

As a whole, ILT is a powerful tool for unraveling the genetic correlation matrix in a natural population, a tool which is still now insufficiently utilized, especially for life-history traits.

\section{Isofemale lines, phenotypic plasticity and reaction norms}

Phenotypic plasticity refers to the fact that a single genotype may produce different phenotypes in different environments (Pigliucci, 2001). In other words, investigations should be carried out on many genetically identical individuals. Clones are easily available in plants but not in animals. In Drosophila, there are two ways for producing identical individuals. One is to investigate the F1 progeny from two isogenic strains (Veuille and Mazeau (1988)). The other is to investigate a parthenogenetic strain, as was recently carried out in $D$. mercatorum (Kristensen et al, 2003).

For D. melanogaster natural population, the ILT is a convenient substitute: within a family, individuals are genetically more similar than between families. The offspring of a given family may be submitted to an environmental gradient (eg different temperatures), so that a phenotypic response curve, the reaction norm, is obtained (David et al, 2004b). Different lines from the same population will exhibit slightly different norms, as illustrated in Figure 4 for wing length. Various genetic correlations may be calculated between family means. For example, we may consider the correlation between the same lines grown at different temperatures (Karan et al, 2000). These correlations are apparently variable, in a complex way, although more extensive data are needed. Correlations can also be considered between sexes and, on average, they are close to 0.80 (Karan et al, 1999a).

As a rule, reaction norms of morphometrical traits with temperature are nonlinear and can be adjusted to quadratic or cubic polynomials. Within the normal thermal range $\left(12-31^{\circ} \mathrm{C}\right.$ in $D$. melanogaster $)$, they exhibit either a regular concave or convex shape, or a sigmoid one.

We illustrate (Figure 5) the average shape of reaction norms for five different traits, wing length, thorax length, ovariole number and sternopleural and abdominal bristle numbers. Interestingly, these curves are all clearly different, both in the position of the maximum and in the relative amplitude of the variations. This diversity is surprising and was not expected. It reflects the complexity of interactions between temperature and developmental genes. Indeed, within the same organ, the wing,

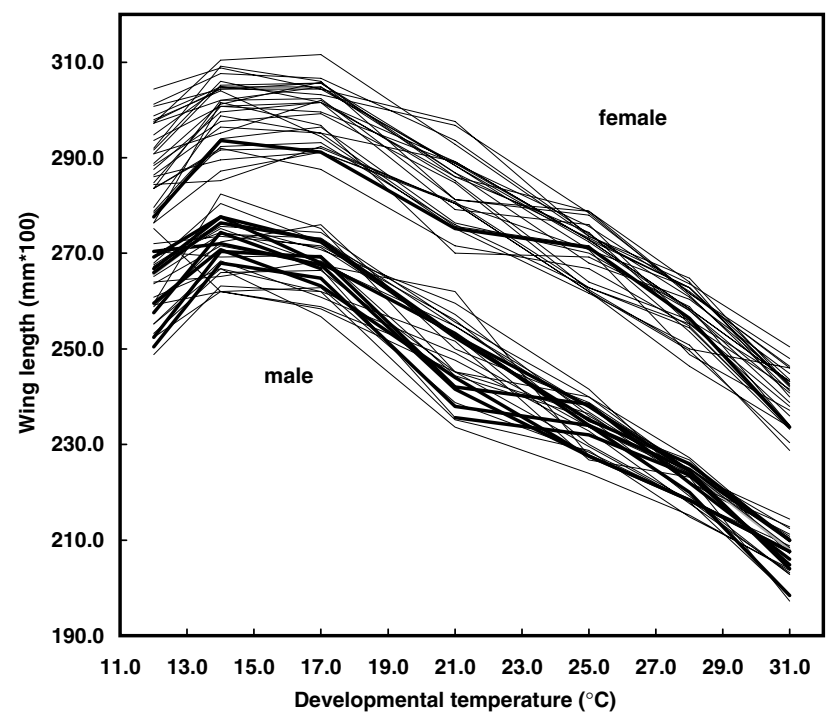

Figure 4 Reaction norms of female and male wing length as a function of growth temperature in 30 isofemale lines from a French (Bordeaux) population. Note the significant size differences among lines. ANOVA (not given) reveals a significant line $*$ temperature interaction, corresponding to the fact that reaction norms are not parallel. 


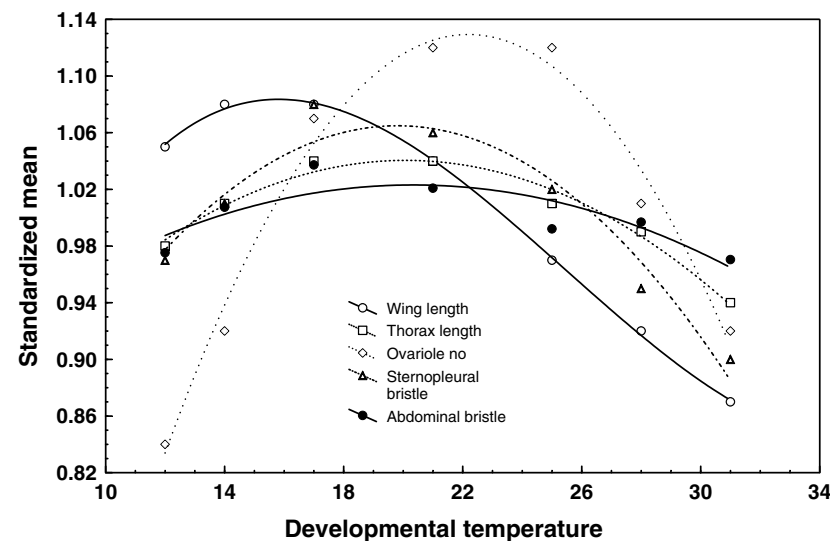

Figure 5 Comparison of the shapes of reaction norms of five different quantitative characters in D. melanogaster females (French population). For comparing the shapes, all curves were standardized to the same overall mean value of 1 . A quadratic polynomial adjustment was used for thorax length, ovariole number and bristle numbers, a cubic polynomial for wing length. Shape variations can be characterized by the temperatures of maximum value, which are 14.4, 19.9, 19.5, 20.4, 22.2 for wing length, thorax length, sternopleural bristle, abdominal bristle and ovariole number, respectively. The magnitude of plasticity may be estimated by calculating a coefficient of variation among temperatures; values are $8.26,3.53,6.32,2.39$ and 10.97 , respectively.

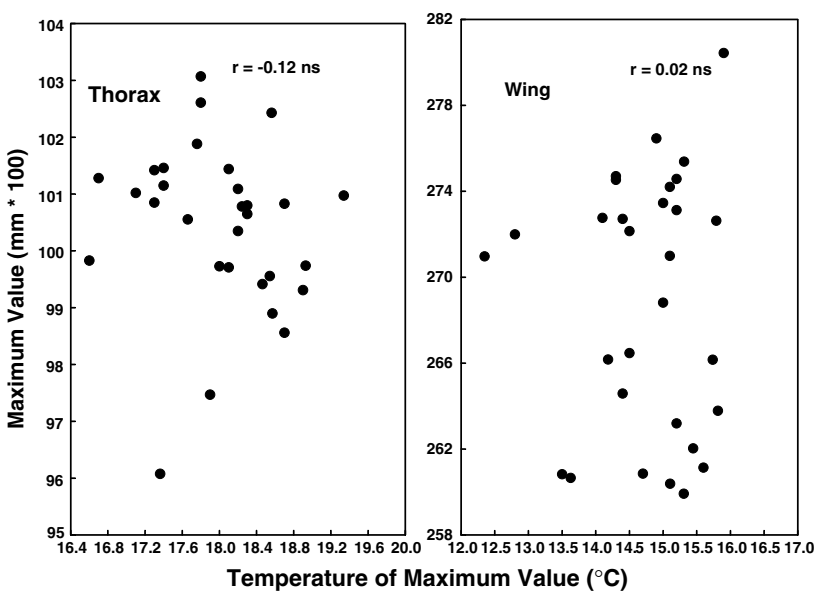

Figure 6 Lack of correlation between size and plasticity of thorax and wing length in females from a French population. Maximum value characterizes the quantitative variability of length among lines. Temperature of maximum value is a plasticity trait.

different parts show very different norms, the main variations being observed along the proximal-distal axis (Moreteau et al, 1998) and not between anterior and posterior compartments.

Phenotypic plasticity is not only expressed in the mean value of a trait, but also in the variability between individuals. Variance parameters are notoriously imprecise so the best information is provided by the withinline variance $(\mathrm{Vw})$ since it is based on a large number of individuals. An interesting conclusion (Delpuech et al, 1995; Noach et al, 1996, Karan et al, 1999a; Moreteau et al, 2003), for traits illustrated in Figure 5, is that when the within-line variability is expressed as a coefficient of variation to eliminate the size effect, a minimum variability is found in the middle of the thermal range, presumably suggesting a physiological optimum. In other words, phenotypic variability exhibits a reaction norm of its own, and, in this case, the curves of different traits have similar shapes.

\section{Characteristic values help to distinguish trait variation and plasticity}

Polynomial adjustments provide, for a given line, an estimate of characteristic values (David et al, 1997). For example, for quadratic concave (ie bowed downwards) curves, as illustrated in Figure 5, the reaction norm may be defined by three values: the coordinates of the maximum and a curvature, the $g_{2}$ parameter. A quadratic function may be written as

$$
y=\mathrm{MV}+g_{2}(t-\mathrm{TMV})^{2}
$$

where MV (the maximum value) is a quantitative property of the trait $y$, while the two other parameters describe the plasticity of the trait. TMV is the temperature of maximum value, $g_{2}$ describes the overall curvature, and $t$ is the growth temperature.

These characteristic values can be calculated for each line, and averaged to define a population or a species. In this respect, significant differences can be found, showing that plasticity may evolve, in an adaptive way, according to environmental temperature (Moreteau et al, 1997; Morin et al, 1997, 1999). However, it is also assumed that variations between lines from the same population have a genetic basis. It is thus possible to calculate genetic correlations. A quite general conclusion is that, for a given trait, mean value and plasticity are not correlated, and this is illustrated for MV and TMV of thorax and wing length (Figure 6). The same conclusion was recently obtained for sternopleural bristle number (Moreteau et al, 2003) in agreement with other investigations (Scheiner and Lyman, 1991; Scheiner, 2002) showing an independence between the dimension and the plasticity of a trait. However, the possible occurrence of specific plasticity genes remains an open question (Via et al, 1995), which might be addressed by using a quantitative trait locus (QTL) design (Dilda and Mackay, 2002).

\section{Sexual dimorphism}

Numerous quantitative traits, which are not directly related to sexuality and reproduction, are expressed differently in males and females. Indeed, recent investigations often suggest different QTL for the same trait in the two sexes (Mackay, 2001). In other words, quantitative trait genes interact with the genetic developmental cascade, which determines sex. Sexual dimorphism (SD) is a special trait since it must be measured on different, although related individuals. In this respect, SD has remained, up to now, poorly investigated, especially from a genetical point of view. There are two main ways to quantify SD: either by considering a difference (eg female-male, F-M), or a ratio (eg female/male, F/M). Since $\mathrm{F}-\mathrm{M}$ is very sensitive to scaling effects, a consensus seems to exist that a ratio should be preferred (Reeve and Fairbairn, 1999; David et al, 2003). For example, the F/M ratios of wing and thorax length are 
almost identical and close to 1.15, while the F-M differences are very different, because wings are much longer than thorax (David et al, 2003).

The ILT provides a direct estimate of dimorphism in each line, and of its mean value in a natural population. The method was also used (David et al, 1994) to analyze its phenotypic plasticity, and it was found that the F/M ratio of size traits increased monotonically but not linearly with increasing growth temperature. The method may also be used to analyze genetic variability among lines and calculate an intraclass correlation (see David et al, 2003). First results suggest that heritability of SD, although significant, is much less than that of the traits measured.

\section{Concluding remarks}

In this review, we have tried to show that ILT, a basic tool for sampling a natural population, can be used for a diversity of purposes in the fields of quantitative and ecological genetics. Such lines permit us to define the mean characteristics of a natural population. With some precautions (genetic repeatability) it can be shown that differences among lines have a genetic basis, which can be estimated by the coefficient of intraclass correlation. However, the relationship between the more usual heritability and the intraclass correlation is not straightforward, presumably because the experimental bottleneck, which is linked to the foundation of each line, unravels a large amount of epistatic interactions, which otherwise would remain cryptic in a large panmictic population. It is thus suggested that we consider the intraclass correlation as a special parameter, called isofemale heritability. However, in addition, isofemale lines can be used in many other types of investigation. They are a powerful means for investigating the genetic correlation matrix in a natural population. They provide a convenient method for the analysis of phenotypic plasticity, the quantitative description of reaction norms and the simultaneous analysis of various traits and of their plasticities. They can also be used for investigating the genetic variability of traits which cannot be measured on the same individual, for example sexual size dimorphism (David et al, 2003).

Other quantitative genetic investigations also use the ILT. For example, for research into QTL (Nuzhdin et al, 1997; Viera et al, 2000), single pairs are used to initiate recombinant inbred (RI) lines. These lines are eventually used to find an association between molecular markers and phenotypes, but they could also be used to calculate an intraclass correlation. A possibility, which remains to be implemented in QTL studies, would be to start from a cross between two extreme lines from different geographic populations. RI lines could be used to analyze the genetic basis of plasticity, and check whether plasticity QTLs are different from trait value QTLs.

The ILT, widespread in Drosophila studies, can be (and indeed is) used in many other species, provided they can be bred, at least for part of their life cycle, under artificial conditions. For example, sawflies (Hymenoptera) are very difficult to rear under laboratory conditions. In nature, however, egg pods, which are the progeny of a single pair, are easily found. This property was used to investigate the genetic variability among different families under different feeding regimes (Kause and Morin, 2001).

Finally, isofemale lines, even in D. melanogaster, are sometimes difficult to collect, especially in remote or exotic places. In temperate countries, another difficulty arises from the fact that natural populations can be sampled only in the warmer months of the year. There is therefore a temptation to keep isofemale lines under laboratory conditions for months or years. As stated above, such lines are prone to genetic drift, possible laboratory adaptation, and also laboratory contamination. For these reasons, we recommend that quantitative characteristics of isofemale lines are investigated in the first or second laboratory generation. After an initial bottleneck, the population size of lines is generally of several tens of flies and, in this respect, a line is akin to a mass population. For this reason, the term isofemale strain is often preferred to isofemale line (Parsons, 1980). Keeping numerous lines from a population is labor intensive and, also, the maintenance of these lines may raise an ethical problem among Drosophila investigators. Most international journals require that any biological material, when published (either DNA sequences, antibodies, laboratory strains or species) should be made available to the international community. The question is: should this rule apply also to lines, kept in laboratories, with all the possible drawbacks underlined above? Our personal experience is that, when possible, a fresh sampling is always better than an investigation of old laboratory lines, especially if we assume that local populations are genetically stable over years (Karan et al, 1999a). The possible phenotypic and genetic stability of local populations is an important problem, which, among many others, deserves more extensive investigations.

\section{References}

Aulard S, David JR, Lemeunier F (2002). Chromosomal inversion polymorphism in tropical populations of Drosophila melanogaster. Genet Res 79: 49-63.

Bell G (1997). The Basics of Selection, Chapman and Hall: NewYork.

Boulétreau J (1978). Ovarian activity and reproductive potential in a natural population of Drosophila melanogaster. Oecologia 35: 319-342.

Capy P, Pla E, David JR (1993). Phenotypic and genetic variability of morphometrical traits in natural populations of Drosophila melanogaster and D. simulans. I. Geographic variations. Genet Sel Evol 25: 517-536.

Capy P, Pla E, David JR (1994). Phenotypic and genetic variability of morphometrical traits in natural populations of Drosophila melanogaster and D. simulans. II. Within population variability. Genet Sel Evol 26: 15-28.

Capy P, Rouault J (1987). Estimation of the allele number in a natural population by the method of isofemale lines. Genetics 117: 795-801.

Coyne JA, Beecham E (1987). Heritability of two morphological characters within and among natural populations of Drosophila melanogaster. Genetics 117: 727-737.

David J, Clavel MF (1965). Interaction entre le génotype et le milieu d'élevage. Conséquences sur les caractéristiques du développement de la Drosophile. Bull Biol Fr Belg 99: 369-378.

David JR, Allemand R, Capy P, Chakir M, Gibert P, Pétavy G et al (2004a). Comparative life histories and ecophysiology of 
Drosophila melanogaster and D. simulans. Genetica 120: 151-163.

David JR, Allemand R, Van Herrewege J, Cohet Y (1983). Ecophysiology: abiotic factors. In: Ashburner M, Carson HL, Thompson JN (eds). Genetics and Biology of Drosophila, Academic Press: New York. pp 105-170.

David JR, Capy P (1988). Genetic variation of Drosophila melanogaster natural populations. Trends Genet 4: 106-111.

David JR, Gibert P, Gravot E, Petavy G, Morin JP, Karan D, Moreteau B (1997). Phenotypic plasticity and developmental temperature in Drosophila: analysis and significance of reaction norms of morphometrical traits. J Therm Biol 22: 441-451.

David JR, Gibert P, Mignon-Grasteau S, Legout H, Pétavy G, Beaumont $C$ et al (2003). Genetic variability of sexual size dimorphism in a natural population of Drosophila melanogaster. J Genet 82: 79-88.

David JR, Gibert P, Moreteau B (2004b). Evolution of reaction norms. Chapter 4. In: TJ DeWitt, SM Scheiner (eds). Phenotypic Plasticity. Functional and Conceptual Approaches, Oxford University Press: NY, USA. pp 50-63.

David JR, Moreteau B, Gauthier JR, Pétavy G, Stockel J, Imasheva A (1994). Reaction norms of size characters in relation to growth temperature in Drosophila melanogaster: an isofemale lines analysis. Genet Sel Evol 26: 229-251.

Delpuech JM, Moreteau B, Chiche J, Pla E, Vouidibio J, David JR (1995). Phenotypic plasticity and reaction norms in temperate and tropical populations of Drosophila melanogaster. Ovarian size and developmental temperatures. Evolution 49: 670-675.

Dilda CL, Mackay TFC (2002). The genetic architecture of Drosophila sensory bristle number. Genetics 162: 1655-1674.

Falconer DS (1989). Introduction to Quantitative Genetics 3rd edn. John Wiley and Sons: New York.

Gibert P, Capy P, Imasheva A, Moreteau B, Morin JP, Pétavy G et al (2004). Comparative analysis of morphological traits among Drosophila melanogaster and D. simulans: genetic variability, clines and phenotypic plasticity. Genetica 120: 165-179.

Gibert P, Moreteau B, David JR (2000). Developmental constraints on an adaptive plasticity: reaction norms of pigmentation in adult segments of Drosophila melanogaster. Evol Dev 2: 249-260.

Gibert P, Moreteau B, Moreteau JC, David JR (1998a). Genetic variability of quantitative traits in Drosophila melanogaster (fruit fly) natural populations: analysis of wild living flies of several laboratory generations. Heredity 80: 326-335.

Gibert P, Moreteau B, Pétavy G, Karan D, David JR (2001). ChillComa tolerance, a major climatic adaptation among Drosophila species. Evolution 55: 1063-1068.

Gibert P, Moreteau B, Scheiner SM, David JR (1998b). Phenotypic plasticity of body pigmentation in Drosophila: correlated variations between segments. Genet Sel Evol 30: 181-194.

Gockel J, Kennington WJ, Hoffmann A, Godstein DB, Partridge L (2001). Nonclinality of molecular variation implicates selection in maintaining a morphological cline of Drosophila melanogaster. Genetics 158: 319-323.

Haerty W, Gibert P, Capy P, Moreteau B, David JR (2003). Microspatial structure of Drosophila melanogaster population in Brazzaville: evidence of natural selection acting on morphometrical traits. Heredity 91: 440-447.

Hoffmann AA, Parsons PA (1988). The analysis of quantitative variation in natural populations with isofemale strains. Genet Sel Evol 20: 87-98.

Imasheva AG, Bubli OA, Lazebny OE (1994). Variation in wing length in Eurasian populations of Drosophila melanogaster. Heredity 72: 508-514.

Imasheva AG, Bosenko DV, Bubli OA (1999). Variation in morphological traits of Drosophila melanogaster (fruit fly) under nutritional stress. Heredity 82: 187-192.
Imasheva AG, Loeschcke V, Zhivotovsky LA, Lazebny OE (1998). Stress temperatures and quantitative variation in Drosophila melanogaster. Heredity 81: 246-253.

Imhof M, Harr B, Brem G, Schlotterer C (1998). Multiple mating in wild Drosophila melanogaster revisited by microsatellite analysis. Mol Ecol 7: 915-917.

Karan D, Morin JP, Gibert P, Moreteau B, Scheiner S, David JR (2000). The genetics of phenotypic plasticity. IX. Genetic architecture, temperature and sex differences in Drosophila melanogaster. Evolution 54: 1035-1040.

Karan D, Morin JP, Gravot E, Moreteau B, David JR (1999b). Body size reaction norms in Drosophila melanogaster: temporal stability and genetic architecture in a natural population. Genet Sel Evol 31: 491-508.

Karan D, Morin JP, Moreteau B, David JR (1998). Body size and developmental temperature in Drosophila melanogaster: analysis of body weight reaction norms. I Therm Biol 23: 301-309.

Karan D, Parkash R, David JR (1999a). Microspatial genetic differentiation for tolerance and utilization of various alcohols and acetic acid in Drosophila species from India. Genetica 105: 249-258.

Kause A, Morin JP (2001). Seasonality and genetic architecture of development time and body size of the birch feeding sawfly Priophorus pallipes. Genet Res 78: 31-40.

Krebs RA, Loeschcke V (1997). Estimating heritability in a threshold trait: heat-shock tolerance in Drosophila buzzatii. Heredity 79: 252-259.

Kristensen TN, Pertoldi C, Andersen DH, Loeschcke V (2003) The use of fluctuating asymmetry and phenotypic variability as indicators of developmental instability: a test of a new method employing clonal organisms and high temperature stress. Evol Ecol Res 5: 53-68.

Lewontin RC (1974). The Genetic Basis of Evolutionary Change, Columbia University Press: New York.

Mackay TF (2001). Quantitative trait loci in Drosophila. Nat Rev Genet 2: 11-20.

Meffert LM (2000). The evolutionary potential of morphology and mating behavior: the role of epistasis in bottlenecked populations. 'Epistasis and the Evolutionary Process'. In: JB Wolf, ED Brodie, MJ Wade (eds). Oxford University Press: Oxford.

Milkman RD, Zeitler RR (1974). Concurrent multiple paternity in natural and laboratory populations of Drosophila melanogaster. Genetics 78: 1191-1193.

Moreteau B, Capy P, Alonso-Moraga A, Munoz-Serrano A, Stockel J, David JR (1995). Genetic characterization of geographic populations using morphometrical traits in Drosophila melanogaster: isogroups versus isofemale lines. Genetica 96: 207-215.

Moreteau B, Gibert P, Delpuech JM, Pétavy G, David JR (2003). Phenotypic plasticity of sternopleural bristle number in temperate and tropical populations of Drosophila melanogaster. Genet Res Camb 81: 25-32.

Moreteau B, Imasheva AG, Morin JP, David JR (1998). Wing shape and developmental temperature in two Drosophila sibling species: different wing regions exhibit different norms of reaction. Russian J Genet 34: 183-192.

Moreteau B, Morin JP, Gibert P, Pétavy G, Pla E, David JR (1997). Evolutionary changes of nonlinear reaction norms according to thermal adaptation: a comparison of two Drosophila species. $C R$ Acad Sci Paris 320: 833-841.

Morin JP, Moreteau B, Pétavy G, David JR (1999). Divergence of reaction norms of size characters between tropical and temperate populations of Drosophila melanogaster and $D$. simulans. I Evol Biol 12: 329-339.

Morin JP, Moreteau B, Pétavy G, Parkash R, David JR (1997). Reaction norms of morphological traits in Drosophila: adaptive changes in a stenotherm circumtropical species. Evolution 51: 1140-1148. 
Noach EK, de Jong G, Scharloo W (1996). Phenotypic plasticity in morphological traits in two populations of Drosophila melanogaster. J Evol Biol 9: 831-844.

Nuzhdin SV, Pasyukova EG, Dilda CL, Zeng ZB, Mackay TFC (1997). Sex-specific quantitative trait loci affecting longevity in Drosophila melanogaster. Proc Natl Acad Sci USA 94: 9734-9739.

Parsons PA (1980). Isofemale strains and evolutionary strategies in natural populations. Evol Biol 13: 175-217.

Parsons PA, Hosgood SMW (1967). Genetic heterogeneity among the founders of laboratory populations of Drosophila. Genetica 38: 328-339.

Partridge L, Hoffmann A, Jones JS (1987). Male size and mating success in Drosophila melanogaster and D. pseudoobscura under field conditions. Anim Behav 35: 468-476.

Pétavy G, David JR, Debat V, Gibert P, Moreteau B (2004). Specific effects of cycling stressful temperatures upon phenotypic and genetic variability of size traits in Drosophila melanogaster. Evol Ecol Res 6: 1-18.

Pigliucci M (2001). Phenotypic Plasticity: Beyond Nature and Nurture, Johns Hopkins University Press: Baltimore.

Reeve JP, Fairbairn DJ (1999). Change in sexual size dimorphism as a correlated response to selection on fecundity. Heredity 83: 697-706.

Rice HS (2002). A general population genetic theory for the evolution of developmental interactions. Proc Natl Acad Sci USA 99: 15518-15523.

Ritchie MG, Kyriacou CP (1994). Genetic variability of courtship song in a population of Drosophila melanogaster. Anim Behav 48: 425-434.
Robertson A (1952). The effect of inbreeding on the variation due to the recessive genes. Genetics 37: 189-207.

Robinson SJW, Partridge L (2001). Temperature and clinal variation in larval growth efficiency in Drosophila melanogaster. J Evol Biol 14: 14-21.

Roff DA, Mousseau TA (1987). Quantitative genetics and fitness: lessons from Drosophila. Heredity 58: 103-118.

Scheiner SM (2002). Selection experiments and the study of phenotypic plasticity. J Evol Biol 15: 89-898.

Scheiner SM, Lyman RF (1991). The genetics of phenotypic plasticity. II. Response to selection. J Evol Biol 4: 23-50.

Simmons LW (2001). Sperm Competition and its Evolutionary Consequences in the Insects. Princeton University press: Princeton and Oxford.

Veuille M, Mazeau S (1988). Genetic variability of sexual behavior in a natural population of Drosophila melanogaster. Behav Genet 18: 389-403.

Via S (1984). The quantitative genetics of polyphagy in an insect herbivore. II. Genetic correlations in larval performance within and among host plants. Evolution 38: 896-905.

Via S, Gomulkiewics R, De Jong G, Scheiner SM, Schlichting CD, van Tienderen PH (1995). Adaptive phenotypic plasticity: consensus and controversy. Trends Evol Ecol 10: 212-217.

Viera C, Pasyukova EG, Zeng S, Hackett JB, Lyman RF, Mackay TFC (2000). Genotype-environment interaction for quantitative trait loci affecting life span in Drosophila melanogaster. Genetics 154: 213-227.

Wolf JB, Brodie ED, Wade MJ (2000). Epistasis and the Evolutionary Process, Oxford University Press: Oxford. 